\title{
Minimal supersymmetric standard model flat direction as a curvaton
}

\author{
Kari Enqvist, ${ }^{1,2}$ Asko Jokinen, ${ }^{2}$ Shinta Kasuya, ${ }^{2}$ and Anupam Mazumdar ${ }^{3}$ \\ ${ }^{1}$ Department of Physical Sciences, P.O. Box 64, FIN-00014, University of Helsinki, Finland \\ ${ }^{2}$ Helsinki Institute of Physics, P.O. Box 64, FIN-00014, University of Helsinki, Finland \\ ${ }^{3}$ Physics Department, McGill University, 3600-University Road, Montreal, Canada H3A 2T8
}

(Received 5 May 2003; published 19 November 2003)

\begin{abstract}
We study in detail the possibility that the flat directions of the minimal supersymmetric standard model (MSSM) could act as a curvaton and generate the observed adiabatic density perturbations. For that the flat direction energy density has to dominate the Universe at the time when it decays. We point out that this is not possible if the inflaton decays into MSSM degrees of freedom. If the inflaton is completely in the hidden sector, its decay products do not couple to the flat direction, and the flat direction curvaton can dominate the energy density. This requires the absence of a Hubble-induced mass for the curvaton, e.g. by virtue of the Heisenberg symmetry. In the case of hidden radiation, $n=9$ is the only admissible direction; for other hidden equations of state, directions with lower $n$ may also dominate. We show that the MSSM curvaton is further constrained severely by the damping of the fluctuations, and as an example, demonstrate that in no-scale supergravity it would fragment into $Q$ balls rather than decay. Damping of fluctuations can be avoided by an initial condition, which for the $n=9$ direction would require an initial curvaton amplitude of $\sim 10^{-2} M_{p}$, thereby providing a working example of the MSSM flat direction curvaton.
\end{abstract}

DOI: 10.1103/PhysRevD.68.103507

PACS number(s): $98.80 . \mathrm{Cq}$

\section{INTRODUCTION}

The minimal supersymmetric standard model (MSSM) is well known to have flat directions, made up of squarks and sleptons, along which the scalar potential vanishes above the soft supersymmetry breaking scale $\sim 1 \mathrm{TeV}[1,2]$. The MSSM flat directions have important cosmological consequences for the early Universe and may seed Affleck-Dine baryo/leptogenesis $[1,3,4]$, give rise to nonthermal generation of supersymmetric dark matter $[5,6]$ or $B$-ball baryogenesis below the electroweak scale [7], and may also act as a source for isocurvature density perturbations [8-10] (for a review, see [11]).

Inflation wipes out all the inhomogeneities along a given flat direction, leaving only the zero mode condensate. However, during inflation quantum fluctuations along the flat directions impart isocurvature density perturbations on the condensate [8]. The isocurvature fluctuations can later be converted into adiabatic perturbations at the time when the flat direction decays into the radiation of the MSSM degrees of freedom [12], provided the flat direction dominates the energy density of the Universe at the time of the decay. Obviously, during inflation the flat direction should be subdominant and its mass should be smaller than the Hubble parameter. This is an example of the so-called curvaton scenario, which in its present incarnation was first discussed in the context of pre-big bang [13] and then applied to ordinary inflation [14]. In many early papers $[14,15]$ the curvaton potential was simply taken as a quadratic potential $V=m^{2} \phi^{2}$.

For the MSSM flat direction curvaton, the potential is determined by the supersymmetry breaking but it is usually dominated by nonrenormalizable operators at large amplitudes. There are three conditions such a potential should satisfy. First, the energy density of the flat direction must contribute negligibly during inflation but should dominate the Universe at later time when the flat direction decays into radiation. Second, the flat direction field should stay in the right place to yield the right amount of (isocurvature) fluctuations. Third, the fluctuations produced during inflation must not die out during the whole process. In [12], we have studied the conditions for the later energy domination, and partly the amplitude of the fluctuations during inflation. Studying the consequences of all these constraints comprehensively is the main purpose of this article.

The structure of the paper is as follows. In Sec. II we discuss general constraints which the flat directions need to obey in order to be viable curvatons. In Sec. III, we follow the dynamics of the flat direction, and study which directions may act as a curvaton. The behavior of the fluctuations is considered in Sec. IV. In Sec. V, we study the MSSM flat direction in no-scale supergravity, and show that $Q$-ball formation is inevitable. Sec. VI is devoted to our conclusions.

\section{FLAT DIRECTION AND INFLATION}

The degeneracy of the effective potential of the MSSM flat direction is lifted by supersymmetry (SUSY) breaking effects and some nonrenormalizable operators. In general, we can thus write the potential as

$$
\begin{gathered}
V(\phi)=\frac{1}{2} m_{\phi}^{2} \phi^{2}+V_{N R}, \\
V_{N R}=\frac{\lambda^{2} \phi^{2(n-1)}}{2^{n-1} M^{2(n-3)}},
\end{gathered}
$$

where $m_{\phi} \sim \mathrm{TeV}$ is the soft SUSY breaking mass, and the flat direction condensate is $\Phi=\phi e^{i \theta} / \sqrt{2} . M$ is the cutoff scale for the low energy effective theory, usually taken to be the Planck scale $M_{p} \simeq 2.4 \times 10^{18} \mathrm{GeV} ; \lambda$ is a coupling constant; and $n=4, \ldots, 9$ is the dimension of the nonrenormalizable 
operator lifting the flat direction, the value of which depends on which particular flat direction one is discussing (for details, see [11]).

In supergravity (SUGRA) theories, the flat direction often acquires the mass of order $H$ because of the SUSY breaking effect due to the finite vacuum energy during inflation [4]. If so, the fluctuation amplitude along the flat direction dies out completely during inflation. We thus demand that the inflation model is such that mass term as large as $H$ is not induced. One example is the SUSY D-term inflation, which during inflation leads to a vanishing Hubble-induced mass term for the flat directions [16]. Another example is models obtained from SUGRA theories with a Heisenberg symmetry on the Kähler manifold [17]. These give rise to a Kähler potential of the form

$$
G=f(\eta)+\ln \left|W\left(\phi_{i}\right)\right|^{2}+g\left(y_{a}\right),
$$

with $\eta=z+z^{*}-\phi_{i}^{*} \phi_{i}$, where $z$ is the Polonyi field, and $\phi_{i}$ and $y_{a}$ are respectively the observable and hidden fields. The latter are defined as the ones that have only Plancksuppressed couplings to the observable sector. In this case there is no mass term in the tree-level potential for the flat direction. No-scale models [18], for which $f(\eta)=-3 \ln \eta$, are a particular realization of the Kähler manifold Heisenberg symmetry. However, even with a Heisenberg symmetry there will be radiatively induced mass squared which is small and negative with $m_{\phi, e f f}^{2} \sim-10^{-2} H^{2}$ [17]. Such a small mass term has only negligible damping effect on the fluctuation amplitude.

In what follows we simply assume that during inflation the flat direction does not get any appreciable Hubbleinduced mass, e.g. by virtue of the Heisenberg symmetry, or by some other reasons.

In order for the curvaton scenario to work, fluctuations of the inflaton should not contribute significantly to the adiabatic density perturbations, so that the Hubble parameter during inflation is $H_{*} \sim \rho_{\text {inf }}^{1 / 2} M_{p}<10^{14} \mathrm{GeV}$. (Needless to say, the energy density of the flat direction should be negligible compared to that of the inflaton, $\rho_{\phi} \ll \rho_{\text {inf }}$.) Then the isocurvature fluctuation of the flat direction is $\delta \phi \sim H_{*} / 2 \pi$. If $\delta \phi / \phi_{*} \sim H_{*} / \phi_{*} \sim 10^{-5}$, where $\phi_{*}$ is the amplitude during inflation, obtained from $V^{\prime \prime}\left(\phi_{*}\right) \sim H_{*}^{2}$, the right amount of density perturbation can be generated provided there is no later damping. A simple analysis shows that during inflation the flat direction field condensate is slow-rolling in the nonrenormalizable potential $V_{N R}$. Thus, the Hubble parameter and the amplitude of the field can respectively be estimated as,

$$
\begin{aligned}
& H_{*} \sim \lambda^{-1 /(n-3)} \delta^{(n-2) /(n-3)} M_{p}, \\
& \phi_{*} \sim \lambda^{-1 /(n-3)} \delta^{1 /(n-3)} M_{p},
\end{aligned}
$$

where $\delta \equiv \delta \phi / \phi_{*} \sim H_{*} / \phi_{*}$.

After inflation, the inflaton ultimately decays into relativistic degrees of freedom. A priori, there are two possibilities. Because the inflaton should give rise to all the observable baryons, conventionally one usually assumes that the inflaton must decay into particles of the observable sector. The other possibility, discussed within the context of the MSSM curvaton scenario [12], is to assume that the inflaton decays into the hidden sector and that the baryons originate solely from the flat direction curvaton decay.

If the inflaton decay products consist of (MS)SM particles, one should consider the behavior of the flat direction in a thermal background which interacts with the condensate field. It has been argued by Postma [19] that the flat direction condensate decays by thermal scattering before its domination. However, in her analysis the thermal decay rate was taken to be $\sim f^{4} T^{2} / m$, whereas in a thermal environment $m$ should be replaced by $f T$ ( $f$ is here some coupling). Nevertheless, the conclusion remains essentially the same, as we now argue. The energy density (amplitude) of the flat direction field in $V \sim T^{2} \phi^{2}$ behaves as $\rho_{\phi} \propto a^{-27 / 8}\left(\phi \propto a^{-21 / 16}\right)$ during the inflaton-oscillation dominated Universe, while $\rho_{\phi} \propto a^{-4}\left(\phi \propto a^{-1}\right)$ during radiation domination. In either case, its energy density decreases not slower than that of radiation, and the amplitude becomes so small that the flat direction condensate cannot dominate the energy density after the zero temperature part $m_{\phi}^{2} \phi^{2}$ becomes important.

These difficulties can be avoided if one takes the inflaton sector to be completely decoupled from the observable one [12]. Indeed, there is not a single realistic particle physics model which would embody the inflaton into the family of the observable fields. In almost all the models the inflaton is a gauge singlet which largely lives in the isolated inflaton sector as if it were part of a hidden world. The coupling of such a singlet to the SM degrees of freedom is usually set by hand. Under such circumstances, perhaps a hidden sector inflaton would be a logical conclusion. Such an inflaton would decay into (light) particles in the hidden sector, but the hidden thermal background would not interact with the flat direction condensate. Note that the reheating of the observable degrees of freedom in the Universe takes place due to the decay of the MSSM flat direction into the MS(SM) degrees of freedom. The curvaton mechanism works successfully if this temperature is high enough. We will come back to this point later.

\section{LATE DOMINATION OF THE FLAT DIRECTION ENERGY DENSITY}

For a successful MSSM curvaton scenario, the energy density of the flat direction condensate should dominate the Universe at the time of its decay. The condensate starts to oscillate when $H \sim m_{\phi}$, and the amplitude at that time is

$$
\phi_{\text {osc }} \sim\left(\frac{m_{\phi} M^{n-3}}{\lambda}\right)^{1 /(n-2)} .
$$

If the reheating by the hidden inflaton occurs earlier, the Universe is dominated by hidden radiation at this time. Since the energy density of the condensate is $\propto a^{-3} \propto H^{-3 / 2}$, we find that 


$$
\begin{aligned}
\left.\rho_{\phi}\right|_{E Q} & \sim m_{\phi}^{2} \phi_{o s c}^{2}\left(\frac{H_{E Q}}{H_{o s c}}\right)^{3 / 2} \\
& \sim\left(m_{\phi} H_{E Q}^{3}\right)^{1 / 2}\left(\frac{m_{\phi} M^{n-3}}{\lambda}\right)^{2 /(n-2)},
\end{aligned}
$$

at the time it equals the hidden radiation density $\rho_{h}$ $\sim H_{E Q}^{2} M_{p}^{2}$. Thus the Hubble parameter at the equality time is

$$
H_{E Q} \sim m_{\phi}\left(\frac{m_{\phi} M^{n-3}}{\lambda M_{p}^{n-2}}\right)^{4 /(n-2)}
$$

In order for the flat direction condensate to have a chance to dominate the Universe, $H_{E Q}$ should be larger than $H$ at the time the curvaton condensate decays. If the decay rate is written as $\Gamma_{\phi} \sim f^{2} m_{\phi}$, where $f$ is some Yukawa or gauge coupling, we have a constraint on the coupling constant which reads

$$
f<\lambda^{-2 /(n-2)}\left(\frac{m_{\phi}}{M_{p}}\right)^{2 /(n-2)}\left(\frac{M}{M_{p}}\right)^{2(n-3) /(n-2)} .
$$

In the opposite case, when the reheating in the hidden sector occurs after the oscillation of the flat direction started, i.e., $H_{o s c}>H_{R H}$, the energy density evolves as

$$
\begin{aligned}
\left.\rho_{\phi}\right|_{E Q} & \left.\sim \rho_{\phi}\right|_{o s c}\left(\frac{H_{R H}}{H_{o s c}}\right)^{2}\left(\frac{H_{E Q}}{H_{R H}}\right)^{3 / 2}, \\
& \sim\left(\frac{m_{\phi} M^{n-3}}{\lambda}\right)^{2 /(n-2)}\left(\frac{T_{R H}^{2}}{M_{p}}\right)^{1 / 2} H_{E Q}^{3 / 2},
\end{aligned}
$$

so that taking into account $H_{o s c}>H_{R H}$ we actually get the same constraint as in Eq. (9).

Even for a small coupling of the order of the electron Yukawa coupling such as $f \sim 10^{-6}$, all $n \leqslant 6$ cases fail to satisfy the condition Eq. (9), whereas $n=7$ is marginal when $\lambda \sim 1$.

The condition Eq. (9) depends on the equation of state of the inflaton decay products in the hidden sector. Let us therefore write the equation of state as $p_{h}=w \rho_{h}$, and assume that the hidden energy has already dominated the Universe when the oscillations along the flat direction begin. Then we obtain the ratio of the energy densities

$$
\begin{aligned}
\frac{\rho_{\phi}}{\rho_{h}} & \left.\sim \frac{\rho_{\phi}}{\rho_{h}}\right|_{o s c}\left(\frac{H}{H_{o s c}}\right)^{-2 w /(1+w)}, \\
& \sim\left(\frac{m_{\phi}}{\lambda M_{p}}\right)^{2 /(n-2)}\left(\frac{H}{H_{o s c}}\right)^{-2 w /(1+w)} .
\end{aligned}
$$

This ratio becomes unity when $H \sim H_{E Q}$. Imposing $H_{E Q}$ $>\Gamma_{\phi} \sim f^{2} m_{\phi}$, we obtain

$$
f<\left[\left(\frac{m_{\phi}}{\lambda M_{p}}\right)^{1 /(n-2)}\right]^{(1+w) / 2 w} .
$$

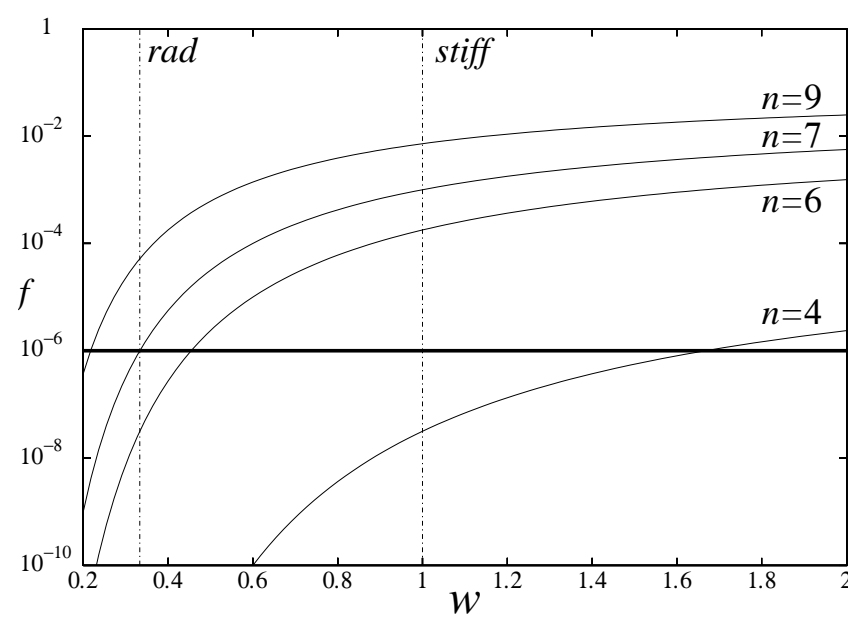

FIG. 1. Constraint on the coupling for $n=4,6,7$, and 9. The allowed region is below these lines and above $f=10^{-6}$.

Notice that this is the same as Eq. (9) for $w=1 / 3$. We show the constraints for $n=4,6,7$, and 9 in Fig. 1. As discussed in [12], the $n=9$ direction is essentially the only viable option for the hidden radiation case, but even $n=6$ directions can be acceptable if the hidden sector fluid has a stiff equation of state $(w=1)$. Notice that $n=4$ directions can never dominate the Universe at any point and are thus completely ruled out as a curvaton candidate.

\section{EVOLUTION OF PERTURBATIONS}

So far we have assumed that the isocurvature perturbation created during inflation does not evolve. This is strictly true in the $m_{\phi}^{2} \phi^{2}$ potential, since both the homogeneous and the (linear) perturbation parts obey the same equations of motion. Here we shall see whether this assumption holds in more general cases.

\section{A. Slow rolling in the nonrenormalizable potential}

For the MSSM curvaton scenario to work, the flat direction must have a vanishing (or more precisely, negligible) mass during inflation. In such a case, the field will be slowrolling in the nonrenormalizable potential $V_{N R}$ $\sim \lambda^{2} \phi^{2(n-1)} / M^{2(n-3)}$. In addition, we assume here that there is no Hubble-induced mass term even after inflation, so that the field will continue slow-rolling in $V_{N R}$ down to the amplitude $\phi_{\text {osc }}$, which is determined by $V_{N R}\left(\phi_{o s c}\right)$ $\sim m_{\phi}^{2} \phi_{o s c}^{2}$. In general, the equations of motion for the homogeneous and fluctuation parts are written respectively as

$$
\ddot{\phi}+3 H \dot{\phi}+V^{\prime}(\phi)=0 \text {, }
$$

$$
\delta \ddot{\phi}_{k}+3 H \delta \dot{\phi}_{k}+\frac{k^{2}}{a^{2}} \delta \phi_{k}+V^{\prime \prime}(\phi) \delta \phi_{k}=0,
$$


where the prime denotes the derivative with respect to $\phi$. Since we are interested only in the super horizon mode ( $k$ $\rightarrow 0$ ), using the slow roll approximation we have

$$
\begin{gathered}
3 H \dot{\phi}+V^{\prime}(\phi)=0, \\
3 H \delta \dot{\phi}+V^{\prime \prime}(\phi) \delta \phi=0 .
\end{gathered}
$$

Hereafter we omit the subscript $k$, understanding that $\delta \phi$ is for the super horizon mode. Then it is easy to obtain the evolution of the ratio of the fluctuation and the homogeneous mode in a $V_{N R} \propto \phi^{2(n-1)}$ potential. The result is

$$
\frac{\delta \phi}{\phi} \sim\left(\frac{\delta \phi}{\phi}\right)_{i}\left(\frac{\phi}{\phi_{i}}\right)^{2(n-2)},
$$

where $i$ denotes the initial values.

During inflation the homogeneous field obeys Eq. (15), which can be easily integrated to yield

$$
\frac{\phi}{\phi_{i}} \simeq\left(1+\frac{1}{3(2 n-3)} \frac{V^{\prime \prime}\left(\phi_{i}\right)}{H^{2}} \Delta N\right)^{-1 / 2(n-2)},
$$

where $\Delta N$ is the number of e-folds. Since we are concerned with the slow-roll regime, it is reasonable to require $V^{\prime \prime}\left(\phi_{i}\right) / H^{2} \lesssim 1$. Hence we have $\phi / \phi_{i} \approx 0.95$ for the last 50 e-folds in the $n=9$ case, for example. This implies that the amplitude of the fluctuation relative to its homogeneous part decreases only by factor $\simeq 2$. Hence during this stage there is essentially no damping. Notice that the slower the condensate field rolls during the last 50 e-folds, the less damping there is.

After inflation the curvaton condensate slow-rolls (albeit marginally), i.e., $V^{\prime \prime}(\phi) \sim H^{2}$, and we can still use the slowroll approximation equations (15) and (16). During this stage, the field amplitude is given by $\phi$ $\sim\left(H M^{n-3} / \lambda\right)^{1 /(n-2)}$, while the Hubble parameter changes from $H_{*}$ to $m_{\phi}$. As a consequence, there is a huge damping given by

$$
\frac{\left(\frac{\delta \phi}{\phi}\right)_{o s c}}{\left(\frac{\delta \phi}{\phi}\right)_{*}} \sim\left(\frac{m_{\phi}}{H_{*}}\right)^{2} \sim \lambda^{2 /(n-3)} \delta^{-2(n-2) /(n-3)}\left(\frac{m_{\phi}}{M}\right)^{2},
$$

where we have used $H_{*} \sim \lambda^{-1 /(n-3)} \delta^{(n-2) /(n-3)} M$ in the last equality, and $\delta \simeq H_{*} / \phi_{*}$ is the fluctuation during inflation. Even for $n=4$, the damping factor is $10^{-10}$ for $M=M_{p}$ and $\delta \sim 10^{-5}$. The situation is still worse for the directions with larger $n$. Hence the primordial fluctuations of the MSSM flat direction curvaton appear to be effectively wiped out. However, before drawing any definite conclusions, one should also consider the effects of Hubble-induced mass terms which can appear after inflation. This is the case, for example, in $D$-term inflation.

\section{B. Positive Hubble-induced mass term}

The Hubble-induced effective potential can be written as

$$
V_{H}=\frac{1}{2} c_{H} H^{2} \phi^{2} .
$$

The sign of the coefficient $c_{H}$ is usually determined by the higher order nonminimal Kähler potential, so there are equal possibilities for positive and negative mass terms. Let us first consider the positive case. When the Hubble-induced effective potential dominates, the equations of motion for the homogeneous and the fluctuation mode have the same form,

$$
\ddot{\psi}+3 H \dot{\psi}+c_{H} H^{2} \psi=0,
$$

where $\psi=\phi$ or $\delta \phi$. From the viewpoint of the evolution of the relative amplitude of the fluctuations, there is no damping. However, the amplitude itself diminishes considerably. If $c_{H}>9 / 16$, the decrease is $\phi \propto H^{1 / 2}$. At the onset of curvaton oscillations, when $H \sim m_{\phi}$, the amplitude of the curvaton is then

$$
\phi_{o s c} \sim \lambda^{-1 / 2(n-3)} \delta^{-(n-4) / 2(n-3)}\left(m_{\phi} M\right)^{1 / 2} .
$$

Since $\delta \geq 10^{-5}$, a maximum is achieved for the largest $n$. For $n=9, \phi_{\text {osc }} \sim 10^{2}\left(m_{\phi} M\right)^{1 / 2}$, which is just $10^{2}$ times larger than in the case of $n=4$, and $\sim 10^{2}$ times smaller than in the slow roll $n=6$ case discussed in the previous subsection. (Notice that the amplitude has the same behavior as in the slow-roll case for $n=4$.) Thus, at the time of its decay, the energy density of the flat direction condensate cannot dominate the Universe.

\section{Negative Hubble-induced mass term}

In this case, the homogeneous field is trapped in the instantaneous minimum $\phi_{m} \sim\left(H M^{n-3} / \lambda\right)^{1 /(n-2)} \propto H^{1 /(n-2)}$. Then, up to a numerical factor, the equation of motion for the fluctuation is identical to the positive Hubble-induced mass case. Hence the amplitude of the fluctuation decreases as $\delta \phi \propto H^{1 / 2}$. Therefore, the ratio of the amplitudes of the homogeneous and the fluctuation modes is given by

$$
\begin{aligned}
\left.\frac{\delta \phi}{\phi}\right|_{\text {osc }} \sim & \left.\frac{\delta \phi}{\phi}\right|_{*}\left(\frac{m_{\phi}}{H_{*}}\right)^{(n-4) / 2(n-2)}, \\
\sim & \lambda^{(n-4) / 2(n-2)(n-3)} \delta^{-(n-4) / 2(n-3)} \\
& \times\left(\frac{m_{\phi}}{M}\right)^{(n-4) / 2(n-2)} .
\end{aligned}
$$

Since the field is released from the trap when $H \sim m_{\phi}$, the subsequent energy domination condition is the same as discussed in the previous section (see Fig. 1). Thus, the only viable direction is $n=9$ (and highly marginally $n=7$ ). We know that for the curvaton scenario $H_{*}<10^{14} \mathrm{GeV}$ so that the fluctuations of the flat direction condensate during inflation cannot be too large: $\delta<3(6) \times 10^{-4}$ for $n=9(7)$. Thus, the conclusion is that ratio of the fluctuation to the homoge- 
neous mode is much less than $10^{-5}$ at the onset of the flat direction condensate oscillations.

One may wonder whether the damping effect becomes any milder for $\left|c_{H}\right|<9 / 16$. Such a situation may be realized in the context of the no-scale SUGRA. However, a small Hubble-induced mass term reduces to the case in Sec. IV A, where the field slow-rolls in a nonrenormalizable potential. Thus, the amplitude of the fluctuations will be wiped out.

\section{The way out}

As seen above, the energy nondomination and/or the damping of the fluctuation amplitude usually kill the MSSM flat direction curvaton scenario. Damping arises because the curvaton has to slide from the slope of the nonrenormalizable potential down to a value at which oscillations commence, a process which takes place slowly and is thus associated with a considerable redshift.

We have found that there are essentially two ways to avoid all these problems. One is that the coupling $\lambda$ of the nonrenormalizable term is small enough so that the potential is effectively of the form $m_{\phi}^{2} \phi^{2}$. In this case, during inflation the amplitude of the flat direction is $\phi_{*} \sim M_{p}$ with $H_{*}$ $\sim 10^{13} \mathrm{GeV}$. The other possibility is that the field amplitude at the end of inflation happens to be of the same order as the amplitude $\phi_{o s c}$. Such a situation may be realized by an extremely long period of inflation, or simply by chance. For the $n=9$ direction, $\phi_{\text {osc }} \sim 10^{16} \mathrm{GeV}$, which is only an order of magnitude less than the "natural" value for $\phi_{*}$. It is conceivable that such a low value of $\phi_{*}$ could be given e.g. by some chaotic initial conditions. Hence we may conclude that hidden inflation with a MSSM curvaton can indeed provide the correct adiabatic density perturbations, although with some difficulty.

\section{FRAGMENTATION OF THE FLAT DIRECTION}

One should also consider the dynamics of the curvaton after its oscillations begin. So far, we have not taken into account the running of the mass of the flat direction. In general, in the gravity mediated SUSY breaking case the mass term in the effective potential can be written as [6]

$$
V(\phi) \simeq m_{\phi}^{2}\left[1+K \log \left(\frac{\phi^{2}}{M^{2}}\right)\right] \phi^{2},
$$

where $K$ is a coefficient obtained from one-loop corrections. MSSM curvaton dynamics is complicated by the fact that when $K$ is negative, the flat direction condensate naturally fragments into $Q$ balls soon after it starts the oscillations $[6,20]$. Then the isocurvature fluctuations of the condensate remain trapped in the $Q$ balls, and will only be released through the decay of the $Q$ balls. They will be converted into adiabatic perturbations only if the energy density of the $Q$ balls dominates the Universe at the time when they decay.

As a concrete example, let us consider no-scale SUGRA. During inflation, the Heisenberg symmetry then guarantees the vanishing of the tree-level Hubble-induced mass. Let us further assume that initially $\phi_{*} \sim \phi_{o s c}$ so that the perturba- tions generated by a curvaton are not damped. $K$ can be computed from the renormalization group equations (RGEs), which to one loop has the form

$$
\frac{\partial m_{i}^{2}}{\partial t}=\sum_{g} a_{i g} m_{g}^{2}-\sum_{a} h_{a}^{2}\left(\sum_{j} b_{i j} m_{j}^{2}+A_{a}^{2}\right),
$$

where $a_{i g}$ and $b_{i j}$ are constants, $m_{g}$ are the gaugino masses, $A_{j}$ the $A$-terms, $h_{a}$ the Yukawa couplings, $t=\ln \left(M_{X} / \mu\right)$ with $M_{X}$ the GUT scale, and $m_{i}$ are the masses of the scalar partners. All the soft SUSY breaking scalar masses vanish at tree-level at the GUT scale except the common gaugino mass $m_{1 / 2}$.

The full renormalization group equations are given in [21]. We neglect all the other Yukawa couplings except the third generation. We assume that the top, bottom and tau Yukawa couplings unify at the GUT scale and normalize the unified coupling through the top quark mass by

$$
m_{\text {top }}\left(m_{\text {top }}\right)=\frac{1}{\sqrt{2}} h_{\text {top }}\left(m_{t o p}\right) v \sin \beta,
$$

where $m_{\text {top }}=174.3 \mathrm{GeV}$ [22], $v=246 \mathrm{GeV}$ and $0<\beta<\pi / 2$ is a free parameter constrained by LEP as $\tan \beta>2.4$ [23]. We find that Yukawa coupling unification does not produce the correct top-Yukawa coupling given by Eq. (26) unless $\tan \beta \geq 2.9$. The unification is actually supported by $\tan \beta$ $\sim 40-50$ [22], so that our calculation clearly covers the relevant range. In [24] $\tan \beta=1$ and only the top-Yukawa coupling was taken into account, which is applicable for small $\tan \beta$.

The mass of the flat direction scalar $\phi$ is the sum of the masses of squark and slepton fields $\phi_{i}$ constituting the flat direction, $m_{\phi}^{2}=\Sigma_{i}\left|p_{i}\right|^{2} m_{i}^{2}$, where $p_{i}$ is the projection of $\phi$ along $\phi_{i}$, and $\Sigma_{i}\left|p_{i}\right|^{2}=1$. The parameter $K$ is then given simply by $[24]$

$$
K=-\left.\frac{1}{2 m_{\phi}^{2}} \frac{\partial m_{\phi}^{2}}{\partial t}\right|_{t=\log \left(M_{X} / \mu\right)} .
$$

To compute $K$, we have to choose the scale $\mu$. The appropriate scale is given by the value of the flat direction field when it begins to oscillate so that $\mu \sim \phi_{\text {osc }}$, see Eq. (6). We have calculated $K$ for two flat directions: $n=7$ direction LLddd (lifted by $H_{u} L L L d d d$ ) and $n=9$ direction QuQue (lifted by $Q u Q u Q u H_{d} e e$ ) [28]. We find that $K$ is generically negative. In Fig. 2 we show the coefficient $K$ plotted against the parameter $\tan \beta$ for the two flat directions with different mixtures of stop, sbottom and stau. In the QuQue direction there are values of $\tan \beta$ where $K$ is positive. This is due to the fact that for $n=9$ the scale of oscillations, $\phi_{\text {osc }} \sim 1.5$ $\times 10^{16} \mathrm{GeV}$, is very close to the GUT scale $M_{X} \sim 3$ $\times 10^{16} \mathrm{GeV}$. For low $\tan \beta$ the Yukawa coupling at the GUT scale is large and dominates over the gaugino terms in the renormalization group equation, driving $\partial m_{i}^{2} / \partial t$ negative and making $K$ positive. When the renormalization group equations are run further, the Yukawa couplings become smaller 

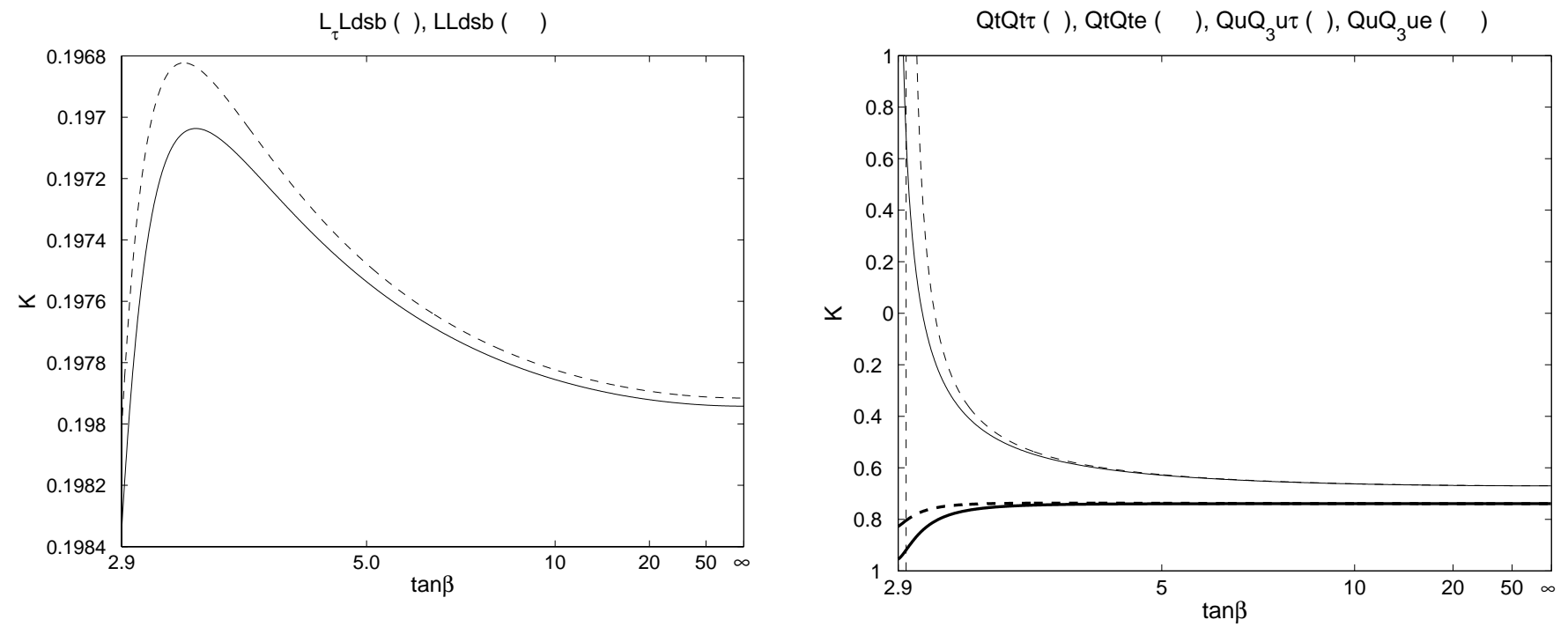

FIG. 2. $K$ vs $\tan \beta$. On the left $L L d s b$ flat direction with a choice of stau in the flat direction (solid line) and no stau in the flat direction (dashed line). On the right $Q u Q u e$ flat direction with $u$ from 3rd generation (thick lines) and $Q$ from 3rd generation (thin lines), $e$ from 3rd generation (solid line) and $e$ from 1 st or 2nd generation (dashed line).

and thus $K$ becomes negative. This is why the $L L d s b$ direction has a negative $K$ for all $\tan \beta$.

Thus in most cases $K<0$ in no-scale SUGRA, so that the flat direction condensates will not decay but instead fragment into lumps which eventually form $Q$ balls. In general, these are long-lived and hence give rise to a reheat temperature which is low (see, e.g., [11]). If R-parity is conserved, decaying $Q$ balls will produce LSPs but with a low reheat temperature, their density might come out to be too high $[6,25]$. The fragmentation of the flat direction condensate is yet another complication for the MSSM curvaton scenario, which we do not attempt to analyze systematically here. The sign of $K$ depends on the running of the RGEs and hence on the initial conditions for the soft SUSY breaking parameters, for which there is no generic form in the class of SUGRA models with a Heisenberg symmetry.

\section{CONCLUSION}

To conclude, an MSSM flat direction curvaton appears to be very much constrained, although not completely ruled out. First of all, we have argued that the constraints depend on the inflaton sector. If the inflaton reheats the Universe with MSSM degrees of freedom, the finite temperature effects on both the effective potential of the flat direction and its decay (or evaporation) process are crucial. As pointed out in Sec. II, the energy density of the flat direction dominated by the thermal mass term cannot overcome the radiation density, so that the curvaton will never dominate the Universe. This seems to exclude flat direction curvatons in the presence of MSSM radiation.

However, if the inflaton is completely in the hidden sector, there will be no thermal corrections to the flat direction. In this case, the curvaton has to provide both the adiabatic density perturbations as well as dark and baryonic matter. The general requirement for this scenario is the energy density dominance by the flat direction condensate at the time of its decay, while during inflation its contribution must be negligible. One ingredient is that during inflation the Hubbleinduced mass term should be negligible, a situation that occurs in SUGRA models with a Heisenberg symmetry.

At large amplitudes the effective potential of the flat direction is dominated by nonrenormalizable terms, and hence it is important to follow the dynamics of both the homogeneous and fluctuation modes during and after inflation. We have found that there is considerable damping of the fluctuations, and in general it is hard to obtain a successful curvaton scenario. Within one particular example of the Heisenberg symmetry, the no-scale model, we have also shown that typically the curvaton may fragment and form $Q$ balls rather than decay directly, which will further complicate matters. Indeed, it is not quite obvious whether $Q$ balls would be a help or a hindrance.

Damping of the fluctuations may however be avoided for a class of initial values for the condensate field after inflation. Perhaps the most promising candidate for the hidden inflation MSSM curvaton would be the $n=9$ QuQue 3rd generation direction with an initial amplitude $\phi_{*}$ $\sim 10^{-2} M_{p}$, based on a SUGRA model such that there is no $Q$-ball formation at least in some parts of the parameter space. In such a case one recovers the hot Universe at the temperature $\sim 10^{5} \mathrm{GeV}$, which is high enough for baryogenesis to occur during the electroweak phase transition [26]. Moreover, the reheat temperature is sufficiently low in order not to create thermal or nonthermal gravitinos [27]. More studies are nevertheless needed to settle the open issues in more detail.

Note added in proof. After the completion of this paper Ref. [29] appeared which also discusses the issue of the damping of the perturbations.

\section{ACKNOWLEDGMENTS}

S.K. is grateful to M. Kawasaki for useful discussions. This work has been partially supported by the Academy of Finland grant 51433. 
[1] M. Dine, L. Randall, and S. Thomas, Nucl. Phys. B458, 291 (1996).

[2] T. Gherghetta, C.F. Kolda, and S.P. Martin, Nucl. Phys. B468, 37 (1996).

[3] I. Affleck and M. Dine, Nucl. Phys. B249, 361 (1985).

[4] M. Dine, L. Randall, and S. Thomas, Phys. Rev. Lett. 75, 398 (1995)

[5] A. Kusenko and M.E. Shaposhnikov, Phys. Lett. B 418, 46 (1998).

[6] K. Enqvist and J. McDonald, Nucl. Phys. B538, 321 (1999).

[7] K. Enqvist and J. McDonald, Phys. Lett. B 425, 309 (1998).

[8] K. Enqvist and J. McDonald, Phys. Rev. Lett. 83, 2510 (1999).

[9] K. Enqvist and J. McDonald, Nucl. Phys. B570, 407 (2000).

[10] M. Kawasaki and F. Takahashi, Phys. Lett. B 516, 388 (2001).

[11] K. Enqvist and A. Mazumdar, Phys. Rep. 380, 99 (2003).

[12] K. Enqvist, S. Kasuya, and A. Mazumdar, Phys. Rev. Lett. 90, 091302 (2003).

[13] K. Enqvist and M.S. Sloth, Nucl. Phys. B626, 395 (2002).

[14] D.H. Lyth and D. Wands, Phys. Lett. B 524, 5 (2002); D.H. Lyth, C. Ungarelli, and D. Wands, Phys. Rev. D 67, 023503 (2003).

[15] T. Moroi and T. Takahashi, Phys. Lett. B 522, 215 (2001); Phys. Rev. D 66, 063501 (2002); N. Bartolo and A.R. Liddle, ibid. 65, 121301 (2002); M.S. Sloth, Nucl. Phys. B656, 239 (2003); K. Dimopoulos and D.H. Lyth, hep-ph/0209180; M. Bastero-Gil, V. Di Clemente, and S.F. King, Phys. Rev. D 67, 103516 (2003); T. Moroi and H. Murayama, Phys. Lett. B 553, 126 (2003); J. McDonald, Phys. Rev. D 68, 043505 (2003).

[16] C.F. Kolda and J. March-Russell, Phys. Rev. D 60, 023504 (1999).
[17] M.K. Gaillard, H. Murayama, and K.A. Olive, Phys. Lett. B 355, 71 (1995).

[18] For a review, see A.B. Lahanas and D.V. Nanopoulos, Phys. Rep. 145, 1 (1987).

[19] M. Postma, Phys. Rev. D 67, 063518 (2003).

[20] S. Kasuya and M. Kawasaki, Phys. Rev. D 61, 041301 (2000); 62, 023512 (2000); S. Kasuya, in Particles, Strings and Cosmology: PASCOS 99, Proceedings of the 7th International Symposium, Lake Tahoe, California, 1999, edited by K. Cheung et al. (World Scientific, Singapore, 2000), p. 301.

[21] K. Inoue, A. Kakuto, H. Komatsu, and S. Takeshita, Prog. Theor. Phys. 68, 927 (1982); 70, 330(E) (1983); 71, 413 (1984).

[22] Particle Data Group, K. Hagiwara et al., Phys. Rev. D 66, 010001 (2002).

[23] LEP Higgs Working Group Collaboration, hep-ex/0107030.

[24] K. Enqvist, A. Jokinen, and J. McDonald, Phys. Lett. B 483, 191 (2000).

[25] M. Fujii and K. Hamaguchi, Phys. Rev. D 66, 083501 (2002).

[26] V.A. Kuzmin, V.A. Rubakov, and M.E. Shaposhnikov, Phys. Lett. B 155, 36 (1985).

[27] J. Ellis, J.E. Kim, and D.V. Nanopoulos, Phys. Lett. 145B, 181 (1984); J. Ellis, D.V. Nanopoulos, K.A. Olive, and S.-J. Rey, Astropart. Phys. 4, 371 (1996); A.L. Maroto and A. Mazumdar, Phys. Rev. Lett. 84, 1655 (2000); R. Kallosh, L. Kofman, A.D. Linde, and A. Van Proeyen, Phys. Rev. D 61, 103503 (2000); R. Allahverdi, M. Bastero-Gil, and A. Mazumdar, ibid. 64, 023516 (2001).

[28] We denote $L L d d d$ by $L L d s b$ since all the family indices in $d d d$ have to be different.

[29] K. Dimopoulos, G. Lazarides, D. Lyth, and R. R. de Austri, Phys. Rev. D (to be published), hep-ph/0308015. 